Cómo citar este artículo: Pérez-Sánchez, A., Alfaro-Martínez, A., Segura-Silva, R. M., Zamora-Sánchez, J., Pérez-González, E., Martínez-Armas, L., Salinas-Rodríguez, D., \& Moreira-Rubio, A. (2020). Evaluación de un tapón de caucho nitrilo como sistema de envase-cierre del inmunógeno Gavac ${ }^{\circledR}$. Rev.investig.desarro.innov., 11 (1), 159-172.

\title{
Evaluación de un tapón de caucho nitrilo como sistema de envase-cierre del inmunógeno Gavac ${ }^{\circledR}$
}

\section{Evaluation of a rubber nitrile stopper as container-closure system of Gavac ${ }^{\circledR}$ vaccine $^{2}$}

\author{
Amaury Pérez-Sánchez ${ }^{1}$ \\ Arlenis Alfaro-Martínez ${ }^{2}$ \\ Rutdali MaríaSegura-Silva ${ }^{3}$ \\ Jesús Zamora-Sánchez ${ }^{4}$ \\ Eikel Pérez-González ${ }^{5}$ \\ Lidiana Martínez-Armas ${ }^{6}$ \\ Diasmarys Salinas-Rodríguez ${ }^{7}$ \\ Alain Moreira-Rubio ${ }^{8}$
}

\begin{abstract}
Resumen
Los sistemas de envase-cierre escogidos, tienen que ser compatibles con el preparado para el cual se aplican a lo largo de su período de validez. En el presente trabajo se realizó la evaluación del tapón de caucho nitrilo (RL8-9-105/0) en sustitución del tapón de caucho acrilonitrilo (FM84/5) como sistema de envase-cierre del inmunógeno Gavac ${ }^{\circledR}$. Se empleó el software estadístico Statgraphics ${ }^{\circledR}$ Plus 5.1, para el procesamiento de los datos. Se llevó a cabo un análisis de la gestión de riesgo mediante un diagrama de causa y efecto, se determinó la conformidad de este tapón con las especificaciones de calidad referidas en el NP 1078, se compararon las características físico-químicas de ambos tapones según las características de calidad del fabricante, se determinó el efecto del placebo sobre las propiedades físicas del tapón y se evaluó el sistema envase-cierre utilizando el tapón de caucho nitrilo mediante un ensayo de hermeticidad y una prueba de esterilidad. Se concluyó que el tapón de caucho nitrilo RL8-9-105/0 puede ser empleado como sistema de envase-cierre del inmunógeno $\mathrm{Gavac}^{\circledR}$, sin que se afecten sus principales parámetros de calidad.
\end{abstract}

Palabras clave: calidad, esterilidad, hermeticidad, inmunógeno Gavacâ.

\begin{abstract}
The chosen container-closure systems must be compatible with the preparation for which they are applied throughout their validity period. In the present work, the evaluation of the nitrile rubber stopper (RL8-9-105 / 0) was carried out in substitution of the acrylonitrile rubber stopper (FM84 / 5) as a container-closure system for the $\mathrm{Gavac}^{\circledR}$ immunogen. The statistical software Statgraphics ${ }^{\circledR}$ Plus 5.1 was used for data processing. A risk management analysis was carried out by means of a cause and effect diagram, the conformity of this cap with the quality specifications referred to in NP 1078 was determined, the physicochemical characteristics of both caps were compared according to the characteristics manufacturer's quality standard, the effect of placebo on the physical properties of the stopper was determined and the containerclosure system using the nitrile rubber stopper was evaluated by means of a tightness test and a sterility test. It was concluded that the RL8-9-105 / 0 nitrile rubber stopper can be used as a container-closure system for the $\mathrm{Gavac}^{\circledR}$ immunogen, without affecting its main quality parameters.
\end{abstract}

Keywords: quality, sterility, impermeability, Gavacâ vaccine.

1 Ingeniero Químico, Estudiante de Maestría en Biotecnología, Universidad de Camagüey, Camagüey, Cuba. E-mail: amaury.perez84@gmail.com. ORCID:0000-0002-0819-6760

2-8 Centro de Ingeniería Genética y Biotecnología de Camagüey, Cuba. 


\section{Introducción}

Debido a los elevados estándares de calidad que se exigen para el acceso a los mercados, las compañías farmacéuticas se deben esforzar para que cada componente del producto final, incluyendo materiales de empaque, cumplan con las especificaciones establecidas durante el ciclo de vida del producto. Tales exigencias imprimen elevada complejidad al aseguramiento estable y con inmediatez de los insumos necesarios para las producciones, que cumplan con los requerimientos de calidad establecidos para las mismas. Esto conlleva a la necesidad de implementar sistemas de evaluación de cambios efectivos que permitan introducir nuevos insumos en las producciones, sin afectar las especificaciones establecidas para cada uno de ellos: esta generalidad se aplica usualmente a los sistemas envase-cierre.

Para agilizar estos procesos de cambio, la Food and Drugs Administration (FDA, 2008), publicó una guía donde incluyó recomendaciones a los fabricantes de productos biológicos, para utilizar las pruebas de integridad durante la sustitución de los ensayos de esterilidad de los sistemas envase-cierre. En dicho análisis, se emplean métodos como el diagrama de causa y efecto, que ayuda a identificar las posibles causas y facilita decisiones que propician una disminución significativa del efecto (Coccia, 2017).

El establecimiento de la seguridad de los sistemas de envase-cierre, es de gran importancia para las industrias: médica, biotecnológica y farmacéutica, con el fin de garantizar la seguridad tanto del producto como del usuario. Los sistemas de envase cierre deben mantener la esterilidad y la calidad de productos estériles, biológicos y vacunas, a lo largo de su tiempo de vida (Ewan et al., 2015), y protegerlos de contaminantes que potencialmente puedan cruzar la barrera establecida, entre los cuales se incluyen: microorganismos, gases reactivos y otras sustancias (Duncan, 2019).
Los sistemas de envase-cierre, están formados por componentes de empaque primarios y secundarios. Los componentes primarios son aquellos que están en contacto directo con el producto, tales como los tapones de caucho, los viales de vidrio o la jeringuilla, mientras que los componentes secundarios son aquellos componentes que son vitales para asegurar un ensamblaje correcto del empaque, tales como las tapas de aluminio localizadas enzima de los tapones (Booth, 2016).

El ensayo de integridad de los sistemas enva- secierre, necesita mostrar la imagen completa de la integridad del envase-cierre a lo largo del tiempo de vida del producto. Esta verificación de la integridad del empaque ocurre típicamente durante tres fases, a saber: el desarrollo y validación del sistema producto-empaque; el sitio de manufactura del producto, y la evaluación de la estabilidad del tiempo de vida del producto comercial (Booth, 2016). Los sistemas de envase-cierre de productos biotecnológicos y farmacéuticos, y sus ensayos de integridad, han sido estudiados y evaluados por diversos autores (Ewan et al., 2015; Booth, 2016; Nietoet al., 2016; Nietoetal., 2018; Duncan, 2019).

El Centro de Ingeniería Genética y Biotecnología (CIGB), de Camagüey, Cuba, no ha estado exento de los problemas relacionados con la adecuada utilización de los sistemas de envase-cierre. En la producción del inmunógeno $\mathrm{Gavac}^{\circledR}$, destinado a combatir la garrapata del ganado bovino Rhipicephalus (Boophilus) Microplus, parásitos que ocasionan grandes pérdidas en las producciones pecuarias (de la Fuente et al., 1999; Canales et al., 2008), se ha utilizado el tapón de caucho acrilonitrilo (FM84/5) de la firma Helvoet ${ }^{\circledR}$ (Helvoet, 2006), aprobado por el sistema de calidad del CIGB (Canales et al., 1997).

En la actualidad, y como parte de su desarrollo tecnológico competitivo, el proveedor Helvoet $^{\circledR}$ comenzó a distribuir en el mercado los tapones de caucho nitrilo (RL8-9-105/0), lo que determinó la realización de un análisis de gestión de riesgo según la guía "ICH: Q9 Quality Risk Management" 
(ICH, 2005), para evaluar el uso de este tapón en el sistema envase-cierre del inmunógeno Gavac ${ }^{\circledR}$. En este trabajo, se lleva a cabo la evaluación técnica del tapón de caucho nitrilo (RL8-9-105/0) para su posible empleo como material de empaque del inmunógeno Gavac ${ }^{\circledR}$.

\section{Materiales y métodos}

2.1 Análisis de la gestión de riesgo de la calidad al emplear el tapón de caucho nitrilo (RL8-9-105/0)
Amaury Pérez-Sánchez, Arlenis Alfaro-Martínez Rutdali María Segura-Silva, Jesús Zamora-Sánchez Eikel Pérez-González, Lidiana Martínez-Armas Diasmarys Salinas-Rodríguez, Alain Moreira-Rubio

como alternativa al tapón de caucho acrilonitrilo (FM84/5)

El análisis de gestión de riesgo de la calidad, implicó efectuar una tormenta de ideas, mediante la cual se enumeraron los posibles riesgos y causas que pueden afectar la calidad del producto al cambiar el tapón. Los resultados se introdujeron en un diagrama causa-efecto (Coccia, 2017), como se ilustra en la figura 1.

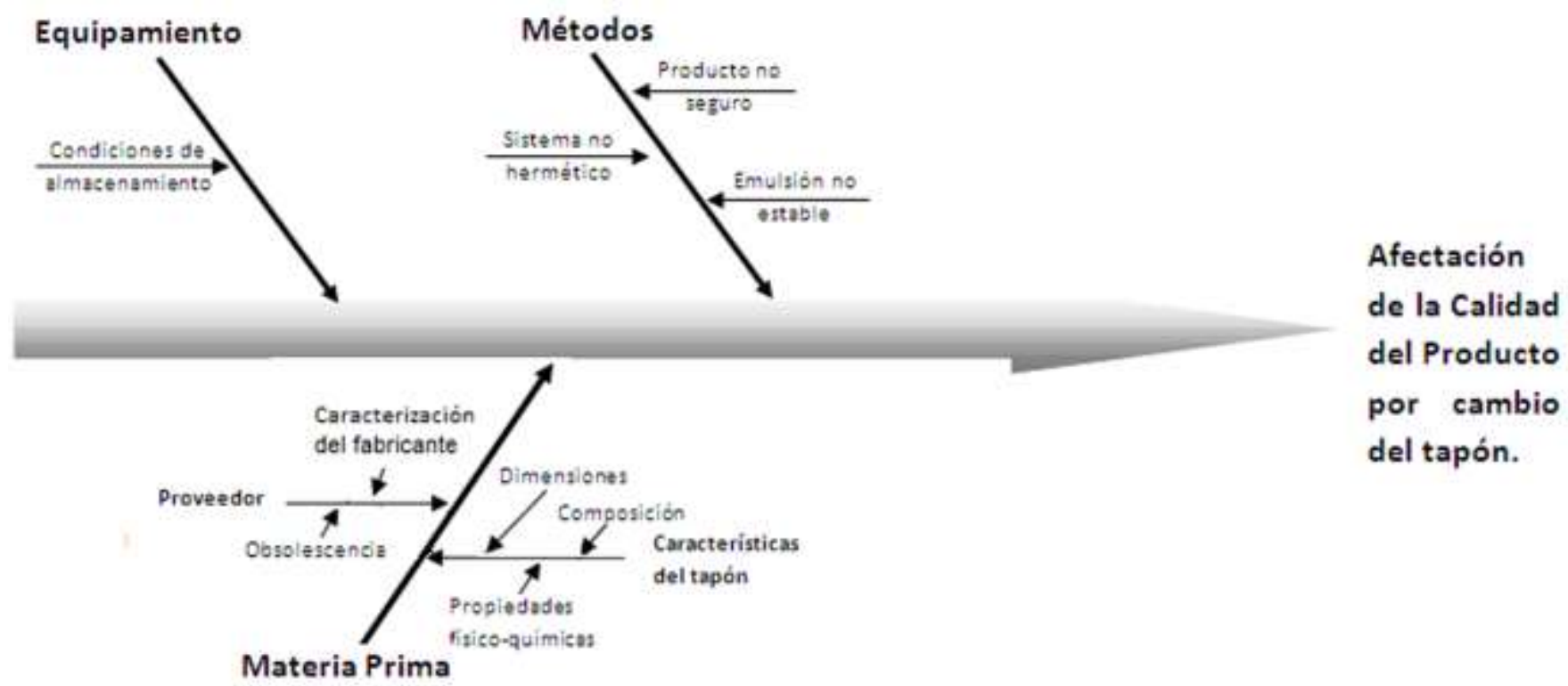

Figura 1. Análisis de posibles causas que pueden afectar la calidad del producto por cambio del tapón.

2.2 Determinación de la conformidad del tapón (RL8-9-105/0) con las especificaciones de calidad definidas en el NP 1078

Se llevaron a cabo todos los ensayos y pruebas incluidas en el Número de Parte (NP) 1078, vigente en el sistema de Aseguramiento de la Calidad del
CIGB, con el fin de verificar si el tapón de caucho nitrilo RL8-9-105/0, cumple con las especificaciones de calidad del tapón de caucho acrilonitrilo (FM84/5) que se utiliza en la actualidad para efectuar el envase del inmunógeno Gavac ${ }^{\circledR}$. La Tabla 1 muestra los ensayos comprendidos en el NP 1078 (CIGB, 1992). 
Tabla 1. Ensayos de calidad establecidos en el NP 1078.

\begin{tabular}{|c|c|}
\hline Ensayo & Límite de aceptación \\
\hline Diámetro del collarín (mm) & $18,8 \pm 0,2$ \\
\hline Espesor del collarín (mm) & $3,3 \pm 0,25$ \\
\hline Diámetro de la falda (mm) & $13,2 \pm 0,1$ \\
\hline Altura total (mm) & $8,8 \pm 0,3$ \\
\hline Opalescencia & No mayor que el patrón tipo II \\
\hline Color & Menos intenso que de la solución de referencia GY 5 \\
\hline Componentes reductores & $<7,0 \mathrm{~mL}$ de $\mathrm{KMnO} 42 \mathrm{mmol}$ en $20 \mathrm{~mL}$ \\
\hline Metales pesados ( $\mathrm{Pb} 2+)$ & $\leq 0,02 \mathrm{mg}$ de $\mathrm{Pb} 2+$ en $10 \mathrm{~mL}$ \\
\hline Iones amonio & $\leq 0,02 \mathrm{mg}$ en $10 \mathrm{~mL}$ \\
\hline Iones cloruro & Máximo 0,04 mg en $10 \mathrm{~mL}$ \\
\hline Alcalinidad o acidez & $\begin{array}{l}<0,3 \mathrm{~mL} \text { de solución de } \mathrm{NaOH} 0,01 \mathrm{~N} \mathrm{o} \mathrm{0,8} \mathrm{mL} \mathrm{de} \mathrm{solución} \mathrm{de} \mathrm{HCl} \\
0,01 \mathrm{~N} \text { en } 20 \mathrm{~mL}\end{array}$ \\
\hline Iones Cinc & $\leq 0,05 \mathrm{mg}$ en $10 \mathrm{~mL}$ \\
\hline Sulfuros volátiles & $\begin{array}{l}\text { La coloración negra del papel de acetato de plomo de la muestra } \\
\text { debe ser menor que la del patrón de } 0,05 \mathrm{mg} \text { de Na2S por cada } \\
20 \mathrm{~cm}^{2} \text { de goma }\end{array}$ \\
\hline U.V. Espectros & D.O. máximo 4 de 220 a 360 nm \\
\hline Fragmentación & Máximo 5 fragmentos en 100 perforaciones \\
\hline Autosellado & Responde \\
\hline
\end{tabular}

2.3 Comparación de las características físico-químicas de los tapones (RL8-9-105/0) y (FM84/5)

Se realizó el análisis de la información técnica suministrada por el proveedor, de los certificados de calidad de los dostapones.

Propiedades químicas: El fabricante realizó los ensayos según la Farmacopea Europea, sección 3.2.9 (EP, 2008), para determinar los siguientes criterios: apariencia, color, materia alcalina, absorción en el espectro ultravioleta (220-360 nm), presencia de sustancias reducidas, concentración de metales pesados, contenido de zinc, amonio, residuos evaporativos, sulfitos y espectro infrarrojo del pirolizado $\left(4000-625 \mathrm{~cm}^{-1}\right)$. Se empleó el agua remanente de la esterilización durante 30 minutos a 121 o $C$ de $100 \mathrm{~cm}^{2}$ de caucho en $200 \mathrm{~mL}$ de agua destilada.
Ensayo de citotoxicidad in vitro: El fabricante determinó la citotoxicidad según ISO 10993-5 (ISO, 2009) y USP 30-NF 25 (Farmacopea, 2007).

2.4 Determinación del efecto del placebo sobre las propiedades físicas del tapón

El placebo, que consiste en una emulsión agua - aceite sin el principio activo del inmunógeno Gavac $^{\circledR}$, se preparó en un vaso precipitado de 1000 $\mathrm{mL}$. Las proporciones de las fases se establecieron según el Procedimiento Patrón de Operación (PPO) 2.14.805.09 (CIGB, 2009). De esta manera, $300 \mathrm{~mL}$ de la fase acuosa se dispersó en $200 \mathrm{~mL}$ de la fase oleosa, utilizando un homogenizador de alta revolución (Ultra Turrax T-50.) de la firma IKA, durante 3 minutos. Luego, para determinar la ocurrencia de variaciones en las características del tapón cuando se ponía en contacto con el placebo bajo condiciones extremas de temperatura, 500 
$\mathrm{mL}$ del mismo se pusieron en contacto con 20 unidades de cada tapón, durante 15 días a una temperatura de $55^{\circ} \mathrm{C}$. Para el ensayo se tomó como control el tapón FM84/5. Para las muestras se tomaron 10 unidades de cada tapón cada dos días hasta el décimo día y el día final del estudio.
Amaury Pérez-Sánchez, Arlenis Alfaro-Martínez Rutdali María Segura-Silva, Jesús Zamora-Sánchez Eikel Pérez-González, Lidiana Martínez-Armas Diasmarys Salinas-Rodríguez, Alain Moreira-Rubio

Se realizó en cada toma de muestra una inspección táctil y visual de los tapones, para verificar que no existieran cambios en la textura y coloración con respecto a los tapones sin tratar. Se midieron el espesor, los diámetros del collarín y de las faldas de los tapones. Las mediciones se efectuaron por triplicado empleando un pie de rey.
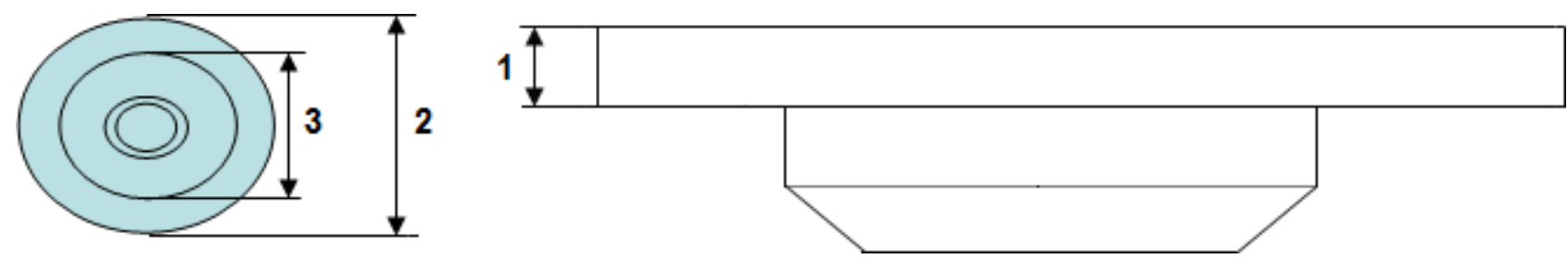

Figura 2. Esquema de los tapones. 1- Espesor, 2- Diámetro del collarín, 3- Diámetro de la falda.

Se efectuó un Análisis de Varianza simple empleando el paquete estadístico Statgraphics ${ }^{\circledR}$ Plus 5.1.

\subsection{Evaluación del sistema envase-cierre}

Se evaluó el sistema envase-cierre, mediante un ensayo de hermeticidad y una prueba de esterilidad.
Ensayo de hermeticidad. Se realizó el ensayo de hermeticidad MGA-0486 (Prueba de sellado para polilaminados de productos higroscópicos, conocida también como prueba de penetración del tinte), de acuerdo con la Farmacopea Europea (EP, 2008). Para ello se llenaron 10 bulbos con agua estéril y se cerraron utilizando los tapones RL8-9-105/0 y sellos Flip-Top, de acuerdo con las características de calidad establecidas en el NP 1317 (CIGB, 2017) (Tabla 2).

Tabla 2. Características de calidad establecidas en el NP 1317.

\begin{tabular}{ll}
\hline Pruebas quese realizan & Límites de aceptación \\
\hline Diámetro interior (mm) & $20,25-20,48$ \\
Espesor de la lámina de aluminio & $0,18-0,22$ \\
Altura de la cápsula de aluminio $(\mathrm{mm})$ & $7,10-7,50$ \\
Diámetro exterior del plástico $(\mathrm{mm})$ & $22,50-23,50$ \\
Altura total (mm) & $8,40-9,20$ \\
Defecto de corte (\%) & Exento y en caso de ocurrir no mayor de un $3 \%$ \\
Contorno de la cápsula de aluminio & Libre de deformaciones oabolladuras \\
Cápsula de aluminio con presencia de lubricantes Libre de lubricantes residuales
\end{tabular}

Los mismos se sumergieron en un vaso precipitado con una solución al $0,1 \%(\mathrm{~m} / \mathrm{v})$ de azul de metileno según indicaciones del PPO 2.31.273.12 (CIGB, 2012). Luego, se colocaron en un horno de vacío a una temperatura de $25^{\circ} \mathrm{C}$, donde se les aplicó vacío lentamente, hasta una presión diferencial de 200 milímetros de mercurio, con respecto al lugar de llenado, por una hora. Después de 5 minutos se insertó una aguja calibre 23 en cada tapón y se procedió con la observación. 
El criterio de aceptación para este ensayo fue la no penetración de la solución en ninguno de los recipientes y que el producto cumpla con las características organolépticas (color blanco brillante, de apariencia homogénea) definidas en las especificaciones de calidad PPO 2.31.249.06 (CIGB, 2006). Se realizó el mismo ensayo con 10 bulbos con tapones de caucho acrilonitrilo (FM84/5) como control.

Prueba de esterilidad. Diez bulbos de polietileno de alta densidad se cerraron con el tapón de caucho nitrilo (RL8-9-105/0) y sellos de fácil apertura y se esterilizaron según PPO 2.14.011.03 (CIGB, 2003) en una autoclave vertical (MEDEXPORT ${ }^{\oplus}$, modelo BKJ-75) a una temperatura de 121 oC durante 30 min. Transcurrido el tiempo de esterilización, los bulbos fueron secados en un horno a 80 o $C$ durante $1 \mathrm{~h}$.

A todos los frascos, se les añadió $100 \mathrm{~mL}$ del inmunógeno Gavac $^{\circledR}$ del lote M05081 según PPO 2.14.801.06 (CIGB, 2006). En este proceso se empleó una llenadora semiautomática (BAUSCH+STROBEL, modelo EDM 520) y una retapadora semiautomática (BAUSCH+STROBEL, modelo HV-100).

Los10bulbosse sometieron aensayo deesterilidad según PPO 4.09.028.92 (CIGB, 1992), en donde estos bulbos se transfirieron a cada uno de los recipientes que contienen suficiente cantidad de Caldo Triptona Soya y Medio de Tioglicolato hasta quedar totalmente cubiertos. A continuación se agita ligeramente. Si no existe aparición de crecimiento microbiano en los medios de cultivo al final de los 14 días, y no se detectó ningún problema en los ensayos de control de calidad a los medios de cultivo utilizados, se dice que la muestra cumple con el ensayo. Por otro lado, si se observa crecimiento microbiano, la muestra no cumple con el ensayo.

\section{Resultados y discusión}

\subsection{Análisis de gestión de riesgo de la calidad}

El diagrama causa - efecto demostró que con el uso del tapón RL8-9-105/0, el producto sigue cumpliendo con las especificaciones de calidad definidas por el sistema de aseguramiento de la calidad deI CIGB. Los aspectos que se abordaron en la tormenta de ideas, se describen a continuación.

\subsection{Caracterización del fabricante}

Helvoet ${ }^{\circledR}$ esun proveedor líder de componentes de envases primarios y secundarios (principalmente de goma y aluminio) en las zonas más avanzadas del mercado farmacéutico parenteral, evaluado por el sistema de calidad CIGB. Tiene una vasta experiencia en la producción de este tipo de productos, bajo estrictos requisitos de calidad. Sus fábricas están certificadas con ISO 9001.

\subsection{Obsolescencia del tapón FM84/5}

El tapón FM84/5, se emplea desde el año 1993 como parte del sistema envase/cierre del inmunógeno Gavac ${ }^{\circledR}$. Asociado a su uso, nunca se han generado no conformidades por el no cumplimiento de las especificaciones de calidad. Helvoet $^{\circledR}$, como parte de su sistema de desarrolloy generación de nuevos productos sustituyó la línea de fabricación del tapón de caucho acrilonitrilo (FM84/5) por un nuevo tapón de caucho nitrilo (RL8-9-105/0) compatible con aceites minerales (Helvoet, 2009).

\subsection{Cumplimiento de las especificaciones de} calidad del tapón caucho nitrilo RL8-9-105/0

Se realizaron los ensayos y pruebas que refiere el NP 1078, para verificar que el nuevo tapón cumpliera con las características de calidad del tapón en uso. Se demostró que el tapón de caucho nitrilo cumple con las especificaciones de calidad definidas, lo cual se muestra en la Tabla 3. 
Tabla 3. Certificación del análisis de calidad de los tapones de caucho nitrilo RL8-9-105/0, analizados en el CIGB.

\begin{tabular}{|c|c|c|}
\hline Análisis realizado & Límite de aceptación & Resultados \\
\hline Absorción UV del extracto & Abs $\leq 4,0$ desde 220 hasta $360 \mathrm{~nm}$ & Pasa la prueba \\
\hline Componentes reductores & Volumen de tiosulfatos $\leq 7,0 \mathrm{~mL}$ & $1,1 \mathrm{~mL}$ \\
\hline Fragmentación & $\leq 5$ fragmentos en 100 perforaciones & 0 \\
\hline Autosellado & $\begin{array}{l}\text { No debe observarse coloración azul en ningún bulbo } \\
\leq 0,05 \mathrm{mg} / 10 \mathrm{~mL}\end{array}$ & 0 \\
\hline Determinación de cinc & $\begin{array}{l}\text { La turbiedad de la muestra no debe ser mayor que la del } \\
\text { patrón }\end{array}$ & Pasa la prueba \\
\hline \multirow[t]{2}{*}{ Acidez o alcalinidad } & $\begin{array}{l}\leq 0,8 \mathrm{~mL} \text { de } \mathrm{HCl} 0,01 \mathrm{M} \\
\leq 0,3 \mathrm{~mL} \text { de } \mathrm{NaOH} \mathrm{0,01} \mathrm{M}\end{array}$ & \multirow[t]{2}{*}{ Pasa la prueba } \\
\hline & $\leq 0,02 \mathrm{mg}$ de $\mathrm{Pb} / 10 \mathrm{~mL}$ & \\
\hline Metales pesados & $\begin{array}{l}\text { La turbiedad de la muestra no debe ser mayor que la del } \\
\text { patrón }\end{array}$ & Pasa la prueba \\
\hline \multirow[t]{2}{*}{ Opalescencia } & $\begin{array}{l}\text { La turbiedad de la muestra no debe ser mayor que la del } \\
\text { patrón }\end{array}$ & \multirow[t]{2}{*}{ Pasa la prueba } \\
\hline & $\leq 0,02 \mathrm{mg}$ de $\mathrm{NH} 4 / 10 \mathrm{~mL}$ & \\
\hline \multirow[t]{2}{*}{ Iones amonio } & $\begin{array}{l}\text { La turbiedad de la muestra no debe ser mayor que la del } \\
\text { patrón }\end{array}$ & \multirow[t]{2}{*}{ Pasa la prueba } \\
\hline & $\leq 0,04 \mathrm{mg}$ de $\mathrm{Cl} / 10 \mathrm{~mL}$ & \\
\hline Iones cloruro & $\begin{array}{l}\text { La turbiedad de la muestra no debe ser mayor que la del } \\
\text { patrón }\end{array}$ & Pasa la prueba \\
\hline
\end{tabular}

3.5 Influencia de la composición del tapón de caucho nitrilo con respecto al caucho acrilonitrilo

A partir de la evaluación realizada según la Farmacopea Europea, sección 3.2.9 (EP, 2008), el fabricante certifica que el tapón RL8-9-105/0, tiene menor cantidad de sustancias reductoras, de materia alcalina, de zinc y menor contenido de residuos evaporativos. Estas características indican que los nuevos tapones podrían tener menos interacción con el producto.
Los tapones RL8-9-105/0 tienen menor contenido de zinc que los tapones de caucho de acrilonitrilo FM84/5. Esto haría muy probable que este material incorpore, de ser así, menos iones Zinc al producto que los tapones en uso. No obstante, en ninguno de los dos casos afectaría la salud del animal, teniendo en cuenta que en humanos la ingesta de este mineral puede llegar hasta los $40 \mathrm{mg} /$ día (Patiño et al., 2011). Las cantidades reportadas por el proveedor para los dos tapones, son: $<0,01$ ppm y $<0.43$ ppm, respectivamente (ver Tabla 4). 
Tabla 4. Propiedades químicas determinadas a los tapones.

\begin{tabular}{llll}
\hline Criterio & Límites & RL8-9-105/0 & FM84/5 \\
\hline Apariencia & Tipo I: 6 & 3,7 & 5,5 \\
Color & Pasa la prueba & Pasa la prueba & Pasa la prueba \\
Materia alcalina $(\mathrm{mL})$ & $0,8(\mathrm{HCl})$ & 0,11 & 0,3 \\
Absorción (220-360 nm) & IIpU I: U,L & 0,04 & - \\
& Tipo II: 4 & - & 4,4 \\
Sustancias reductoras & IIpO I: 5 & 0,62 & - \\
& Tipo II: 7 & - & 6,5 \\
Metales pesados (ppm) & 2 ppm Pb 2+ & $<2$ & $<2$ \\
Zinc (ppm) & 5 ppm Zn 2+ & $<0,01$ & 0,43 \\
Amonio (ppm) & 2 ppm (NH4)+ & $<2$ & $<2$ \\
Residuos de evaporación $(\mathrm{mg})$ & Tipo I: 2 & 0,2 & 1,8 \\
Sulfitos $(\mathrm{ppm})$ & 0,02 & $<0,02$ & $<0,02$ \\
\hline
\end{tabular}

En la Figura 3 se muestran los espectros de absorción (220-360 nm) del extracto acuoso de los tapones FM84/5 y RL8-9-105/0. En esta región es donde absorben las sustancias orgánicas aromáticas. Para el caso del tapón FM84/5 se

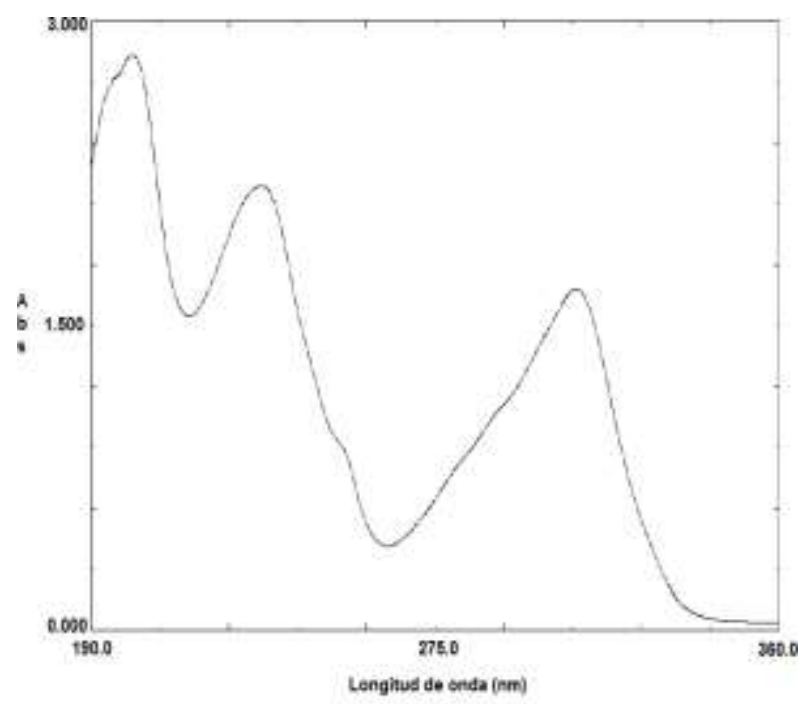

(a) interpreta que durantela extracción acuosa ocurre solubilización de sustancias orgánicas aromáticas, efecto que no se observó en el espectro del tapón RL8-9-105/0.

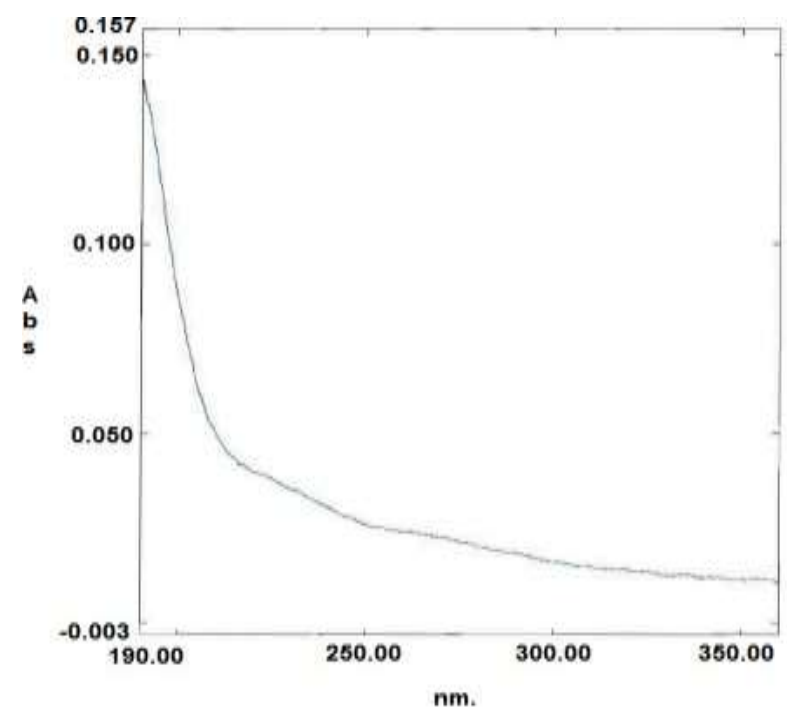

(b)

Figura 3. Espectro de adsorción (220-360 nm) de extracto acuoso según Farmacopea Europea, sección 3.2.9 (EP, 2008) (A) Tapón FM84/5. (B) Tapón RL8-9-105/0.

En la Figura 4 se exponen los espectros infrarrojos de los pirolizados de cada tapón. La región barrida (4000 a $625 \mathrm{~cm}^{-1}$ ) es la utilizada en química orgánica para el estudio estructural de 
las moléculas. Se aprecia que existe coincidencia en la composición orgánica de los tapones; y en cuanto a las cantidades de cada sustancia, existe
Amaury Pérez-Sánchez, Arlenis Alfaro-Martínez Rutdali María Segura-Silva, Jesús Zamora-Sánchez Eikel Pérez-González, Lidiana Martínez-Armas Diasmarys Salinas-Rodríguez, Alain Moreira-Rubio una menor relación para algunas de ellas en el tapón RL8-9-105/0.

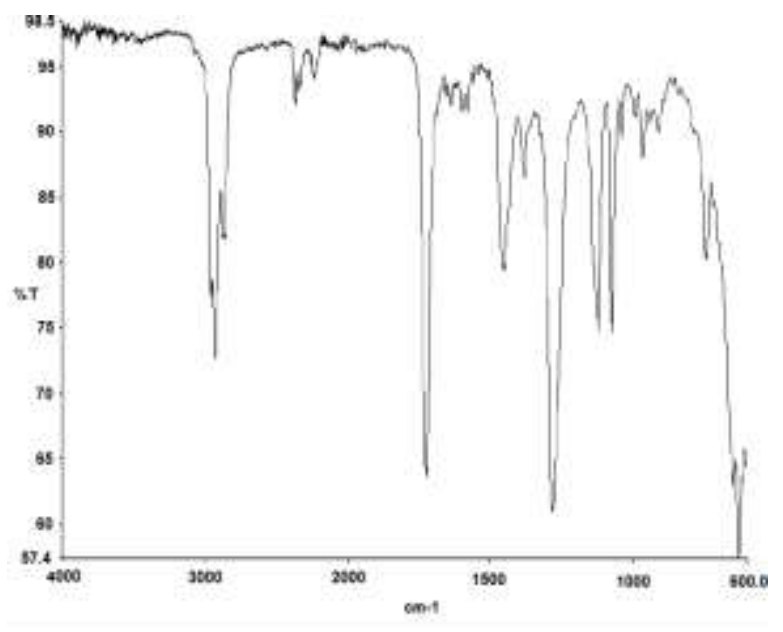

(a)

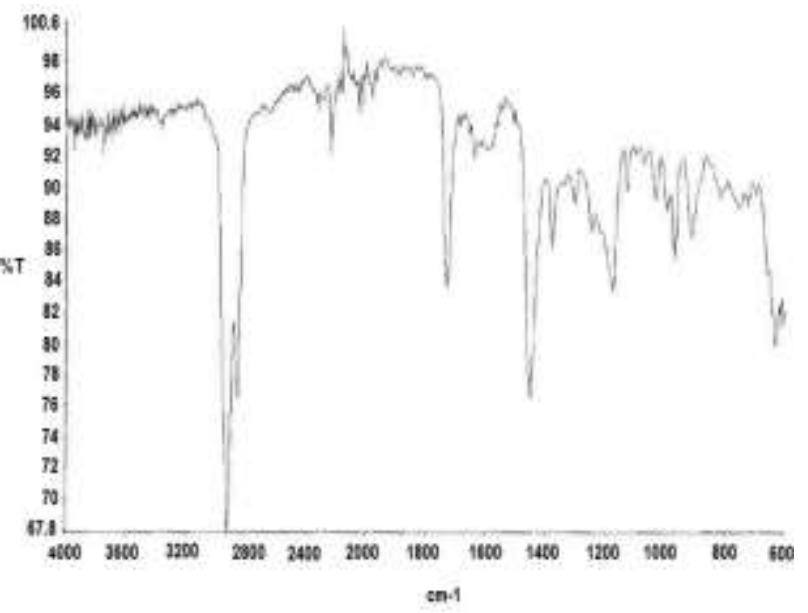

(b)

Figura 4. Espectro infrarrojo del tapón pirolizado $\left(4000-625 \mathrm{~cm}^{-1}\right)$. (A) Tapón FM84/5. (B) Tapón RL8-9-105/0.

La comparación e interpretación de los espectros de adsorción (220-360 nm) e infrarrojo (4000$625 \mathrm{~cm}^{-1}$ ) es muy importante para entender que el tapón RL8-9-105/0 posee una composición química orgánica similar que el tapón FM84/5, incluso en varias sustancias su relación es menor. Teniendo en cuenta lo anterior, es de esperar que la interacción tapón e inmunógeno $\mathrm{Gavac}^{\circledR}$ sea, al menos, similar y por tanto no se alteraría la calidad del producto.
3.6 Interacción/comportamiento ante el placebo del inmunógeno Gavac ${ }^{\circledR}$

En la Tabla 5, se muestran los resultados del análisis estadístico realizado con respecto a las mediciones de: diámetro, espesor del collarín y diámetro de la falda para los tapones RL8-9-105/0 y FM84/5. La Figura 5 muestra los gráficos de cajas y bigotes, obtenidos mediante el software Statgraphics ${ }^{\circledR}$ Plus 5.1, para cada una de las mediciones realizadas.

Tabla 5. Resultados estadísticos sobre las mediciones de: diámetro, espesor del collarín y diámetro de la falda para los tapones RL 8-9-105/0 y FM 84/5.

\begin{tabular}{lllllll}
\hline \multicolumn{1}{c}{ Parámetro } & \multicolumn{1}{c}{$\begin{array}{c}\text { Espesor } \\
\text { collarín } \\
\text { acrilonitrilo }\end{array}$} & $\begin{array}{c}\text { Espesor } \\
\text { collarín } \\
\text { nitrilo }\end{array}$ & $\begin{array}{c}\text { Diámetro } \\
\text { collarín } \\
\text { acrilonitrilo }\end{array}$ & $\begin{array}{c}\text { Diámetro } \\
\text { collarín } \\
\text { nitrilo }\end{array}$ & $\begin{array}{c}\text { Diámetro } \\
\text { falda } \\
\text { acrilonitrilo }\end{array}$ & $\begin{array}{c}\text { Diámetro } \\
\text { falda nitrilo }\end{array}$ \\
\hline Media & 3,37762 & 3,50476 & 18,6452 & 18,7571 & 13,1952 & 13,2024 \\
Varianza & 0,0072 & 0,0019 & 0,0034 & 0,0058 & 0,0022 & 0,0038 \\
Desv. Típica & 0,0854 & 0,0444 & 0,0589 & 0,0762 & 0,0471 & 0,0622 \\
Mínimo & 3,2 & 3,4 & 18,6 & 18,6 & 13,15 & 13,10 \\
Máximo & 3,5 & 3,55 & 18,75 & 18,85 & 13,3 & 13,3 \\
Cociente F & 0,90 & 0,74 & 1,90 & 0,34 & 0,78 & 0,85 \\
Valor P & 0,5214 & 0,6260 & 0,1514 & 0,9049 & 0,5969 & 0,5512 \\
\hline
\end{tabular}




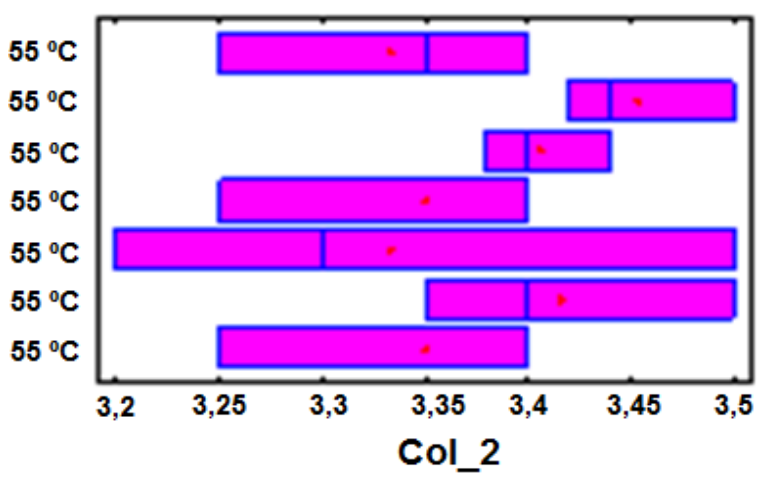

(a)

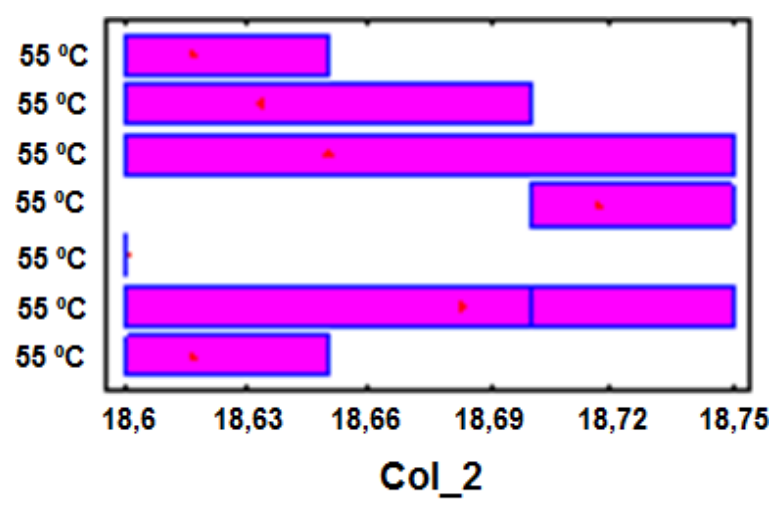

(c)

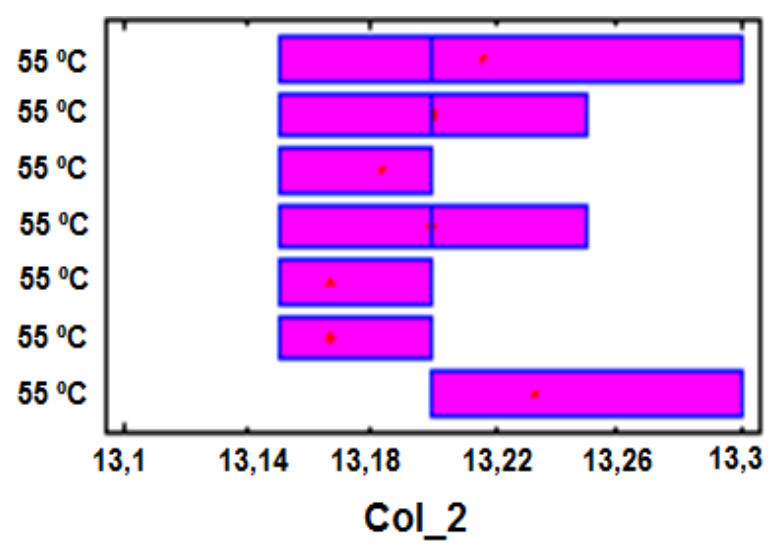

(e)

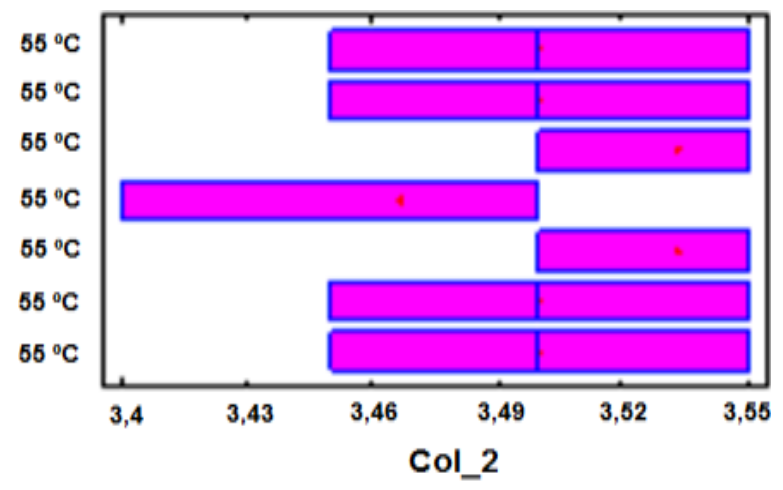

(b)

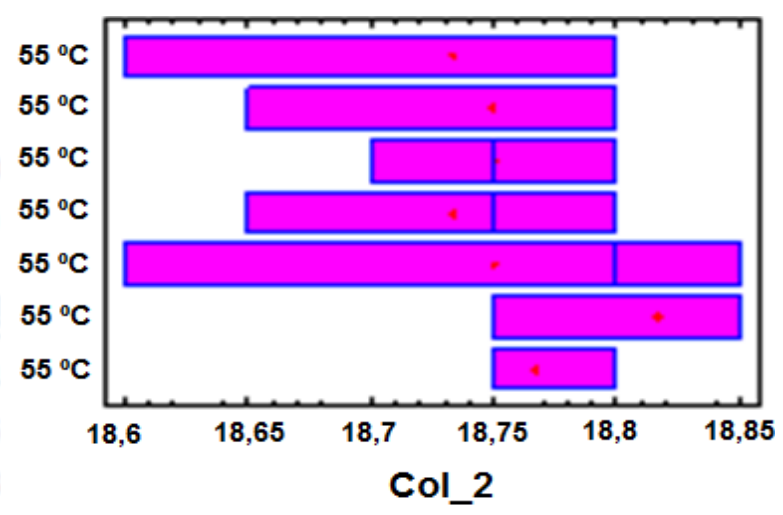

(d)

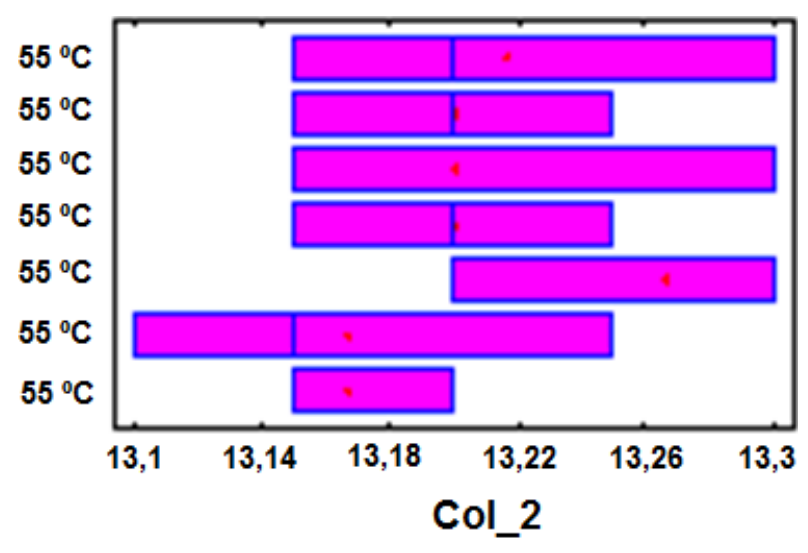

(f)

Figura 5. Gráficos de cajas y bigotes para cada una de las mediciones realizadas. A) Espesor del collarín tapón acrilonitrilo. B) Espesor del collarín tapón nitrilo. C) Diámetro del collarín tapón acrilonitrilo. D) Diámetro del collarín tapón nitrilo. E) Diámetro de la falda tapón acrilonitrilo. F) Diámetro de la falda tapón nitrilo. 
El resultado arrojó que las medidas dimensionales del espesor, el diámetro del collarín y del diámetro de las faldas de los tapones, no difieren $(P>0,05)$. En todos los casos los parámetros evaluados cumplieron con las especificaciones descritas en el NP 1078. Con los tapones ensayados se realizaron pruebas de cierre del frasco de Gavac $^{\circledR}$ y visualmente se comportó similar con una muestra de tapones no sometidos a tratamiento. La comprobación de la compatibilidad con el aceite mineral, es esencial para ser utilizado en el sistema envase-cierre del inmunógeno Gavac $^{\circledR}$. Luego de mantener el tapón RL8-9-105 y el tapón FM84/5 sumergido en placebo, para determinar la ocurrencia de variaciones en las características del tapón cuando se puso en contacto con el placebo.

\subsection{Posible influencia en la hermeticidad del} sistema envase/cierre del Inmunógeno Gavac $^{\circledR}$

Teniendo en cuenta que se trata del mismo proveedor y que las dimensiones de los tapones
Amaury Pérez-Sánchez, Arlenis Alfaro-Martínez Rutdali María Segura-Silva, Jesús Zamora-Sánchez Eikel Pérez-González, Lidiana Martínez-Armas Diasmarys Salinas-Rodríguez, Alain Moreira-Rubio

de caucho RL8-9-105/0 cumplen con el NP 1078, existe un bajo riesgo que falle la hermeticidad del sistema envase-cierre. Los resultados obtenidos en el ensayo de esterilidad utilizando los tapones RL8-9-105/0, indican que pasa la prueba, lo cual significa que existe ausencia de microorganismos viables o potencialmente viables en la muestra. De manera indirecta se confirmó que el sistema envase-cierre cumple su objetivo.

El ensayo de hermeticidad, demostró que el sistema envase-cierre cumple el objetivo previsto en el diseño, porque no hubo incorporación de azul de metileno al interior de los frascos al emplearse los dos tapones en estudio. En la Figura 6 se observa el resultado del ensayo, en el cual se eliminó el sello en los frascos evaluados para visualizar diferencias entre los tapones.

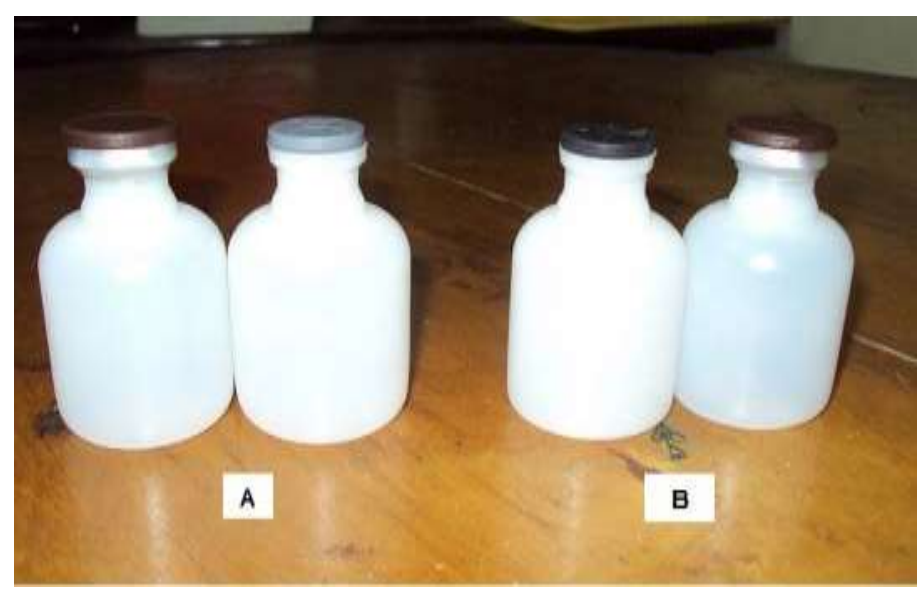

Figura 6. Imágenes del resultado de la prueba de hermeticidad del sistema envase-cierre con azul de metileno.(A)-Tapón RL8-9-105/0, izquierda: control positivo, derecha: muestra de frascosensayados;

(B)-Tapón FM84/5, izquierda: muestra de frascos ensayados, derecha: control positivo.

\subsection{Posible influencia en la toxicidad: Seguridad}

El ensayo de toxicidad in vitro, realizado a los tapones RL8-9-105/0 según ISO 10993 (ISO, 2009) y USP 30-NF 25 (Farmacopea, 2007), mostró que la muestra procesada resultó no citotóxica (frente a células de mamíferos) después de $48 \mathrm{~h}$ de observación. Tanto el control negativo como el positivo evidenciaron la validez del ensayo biológico realizado, dando una garantía al uso de los tapones, lo cual quedó demostrado sobre la base de los criterios de evaluación mencionados, 
concluyendo que el producto de ensayo se considera no citotóxico.

\subsection{Aseguramiento del producto $\mathrm{Gavac}^{\circledR}$ al distri- buirse con el tapón RL8-9-105/0}

EI CIGB implementará un monitoreo de todos los lotes distribuidos durante el período de evaluación deeste cambio. Esto permitirá de igual manera que exista un control y vigilancia de la implementación del programa de control integrado y se emitan alertas ante cualquier anomalía. En función de todo lo anterior es de esperar que el riesgo que se asumirá con la sustitución del tapón FM84/5 por el RL8-9-105/0, se minimizará con un seguimiento, incluso después de la aplicación del inmunógeno. De estamanera se recomienda desarrollaryaplicar un estudio semejante al mostrado en este trabajo, en caso de que el proveedor vuelva a modificar el tipo de tapón en elfuturo.

\section{Conclusiones}

El tapón de caucho nitrilo RL8-9-105/0, cumple con todas las especificaciones de calidad definidas en el NP 1078.

El tapón de caucho nitrilo RL8-9-105/0 contiene menor cantidad de sustancias reductoras, de materia alcalina, de zinc y menor contenido de residuos evaporativos, lo cual indica que su interacción con el inmunógeno Gavacâ será, al menos, similar que el tapón de acrilonitrilo FM84/5, por lo que no se alteraría la calidad del producto.

Las dimensiones de: espesor, diámetro del collarín y diámetro de las faldas de ambos tapones evaluados, no difieren entre sí, cumpliendo en todos los casos con las normas de calidad establecidas en el NP 1078.

Los resultados del ensayo de esterilidad realizado a los tapones RL8-9-105/0, arrojaron que pasa la prueba, lo cual significa que existe ausencia de microorganismos viables o potencialmente viables en la muestra, por lo que se establece que el sistema envase-cierre cumple su objetivo.

El ensayo de toxicidad in vitro realizado a los tapones RL8-9-105/0, demostró que la muestra procesada resultó no citotóxica después de 48 $\mathrm{h}$ de observación, por lo que se declara que el producto bajo ensayo se considera no citotóxico.

El tapón RL8-9-105/0 tiene características físicas, químicas y dimensionales adecuadas para ser empleado en el sistema envase-cierre del Inmunógeno $\operatorname{Gavac}^{\circledR}$, concluyendo que el riesgo de falla es nulo.

\section{Referencias}

Booth, C. (2016). Understanding Container Closure Integrity Testing. Recuperado de: https://www. americanpharmaceuticalreview.com/Featured-

Articles/239498-Understanding- ContainerClosure-Integrity-Testing/

Canales, M., Enriquez, A., Ramos, E., Cabrera, D., Dandie, H., Soto, A., Falcón, V., Rodríguez, M., \& de la Fuente, J. (1997). Large-scale production in Pichia pastoris of the recombinant vaccine GavacTM against cattle tick. Vaccine, 15 (4), 414-422. doi: 10.1016/S0264-410X(96)00192-2.

Canales, M., Pérez, J. M., Naranjo, V., Nijhof, A. M., Hope, M., Jongejan, F., \& de la Fuente, J. (2008). Expression of recombinant Rhipicephalus (Boophilus) microplus, R. annulatus and R. decoloratus Bm86 orthologs as secreted proteins in Pichia pastoris. BMC Biotechnology, 8 (14), 1-12. doi: 10.1186/1472-6750-8-14.

Centro de Ingeniería Genética y Biotecnología, CIGB. (1992). Ensayo de esterilidad (Método directo) (PPO 4.09.028.92). Camagüey, Cuba: CIGB.

Centro de Ingeniería Genética y Biotecnología, CIGB. (1992). Tapón de caucho acrilonitrilo, negro, 20 mm, tipo II (NP 1078). Camagüey, Cuba. CIGB. 
Centro de Ingeniería Genética y Biotecnología, CIGB. (2003). Preparación de los materiales utilizados en la filtración esterilizante del IFA (PPO 2.14.011.03). Camagüey, Cuba: CIGB.

Centro de Ingeniería Genética y Biotecnología, CIGB. (2006). Determinación de Características Organolépticas a emulsiones oleosas (PPO 2.31.249.06). Camagüey, Cuba. CIGB.

Centro de Ingeniería Genética y Biotecnología, CIGB. (2006). Llenado y retapado del Inmunógeno Gavac $^{\circledR}$ utilizando la llenadora modelo EDM 520 y la retapadora modelo HV-100, ambas de la firma BAUSCH+STROBEL. (PPO2.14.801.06). Camagüey, Cuba: CIGB.

Centro de Ingeniería Genética y Biotecnología, CIGB. (2009). Formulación y llenado de los lotes placebo de Gavac $^{\circledR}$ (PPO 2.14.805.09). Camagüey, Cuba: CIGB.

Centro de Ingeniería Genética y Biotecnología, CIGB. (2012). Ensayo de hermeticidad en sistemas de envase-cierre (PPO 2.31.273.12). Camagüey, Cuba: CIGB.

Centro de Ingeniería Genética y Biotecnología, CIGB. (2017). Sello de fácil apertura de aluminio y plástico color carmelita, $20 \mathrm{~mm}$ (NP 1317). La Habana, Cuba: CIGB.

Coccia, M. (2017). The Fishbone diagram to identify, systematize and analyze the sources of general purpose technologies. Journal of Social and Administrative Sciences, 4(4), 291-303. doi: 10.1453/jsas.v4i4.1518.

De la Fuente, J., Rodríguez, M., Montero, C., Redondo, M., García-García, J. C., Méndez, L., Serrano, E., Valdés, M., Enríquez, A., Canales, M., Ramos, E., Boué, O., Machado, H., \& Lleonart, R. (1999). Vaccination against ticks (Boophilus spp.): the experience with the Bm86-based vaccine
Amaury Pérez-Sánchez, Arlenis Alfaro-Martínez Rutdali María Segura-Silva, Jesús Zamora-Sánchez Eikel Pérez-González, Lidiana Martínez-Armas Diasmarys Salinas-Rodríguez, Alain Moreira-Rubio

Gavac $^{\mathrm{TM}}$. GeneticAnalysis:BiomolecularEngineering, 15, 143-148. doi: 10.1016/s1050-3862(99)00018-2.

Duncan, D. (2019). Container Closure Integrity Testing of Finished Sterile Injectable Product. Pharmaceutical Technology, 43(9), 38-41.

European Pharmacopoeia, EP. (2008). 3.2.9. Rubber Closures for Containers for Aqueous Parenteral Preparations, for Powders and for Freeze-Dried Powders (01/2008:30209): European Pharmacopoeia 7.0.

Ewan, S., Jiang, M., Stevenson, C., Henderson, O., Klohr, S., Wessel, M., Mehta, P., Adler, S., Lake, C., Walsh, J., Polomene, T., \& Everaert, V. (2015). Container Closure Integrity Control versus Integrity Testing during Routine Manufacturing. PDA Journal of Pharmaceutical Science and Technology, 69(3), 461-465. doi:10.5731/pdajpst.2015.01071

Farmacopea. (2007). Farmacopea de los Estados Unidos de América USP 30-NF 25 (Vol. 1). Rockville, MD: The United States Pharmacopeial Convention.

Food and Drug Administration, FDA. (2008). Guidance for Industry: Container and Closure System Integrity Testing in Lieu of Sterility Testing as a Component of the Stability Protocol for Sterile Products. Rockville, MD: FDA.

Helvoet. (2006). Compound Data Sheet FM84/5 Black Stopper, 3. Alken, Belgium: Helvoet Pharma.

Helvoet. (2009). Compound Data Sheet RL8-9105/0 Grey Stopper 4. Alken, Belgium: Helvoet Pharma.

International Conference on Harmonisation, $\mathrm{ICH}$. (2005). ICH guideline Q9 on quality risk management. London: European Medicines Agency.

International Organization for Standardization, ISO. (2009). Biological evaluation of medical 
devices - Part 5: Tests for in vitro cytotoxicity 10993-5. Geneva, Switzerland: ISO.

Nieto, A., Roehl, H., Adler, M., \& Mohl, S. (2018). Evaluation of Container Closure System Integrity for Storage of Frozen Drug Products: Impact of Capping Force and Transportation. PDA Journal of Pharmaceutical Science and Technology, 72(6), 544-552. doi: 10.5731/pdajpst.2017.008383.

Nieto, A., Roehl, H., Brown, H., Nikoloff, J., Adler, M., \& Mahler, H.-C. (2016). Evaluation of Container Closure System Integrity for Frozen Storage Drug Products. PDA Journal of Pharmaceutical Science and Technology, 70(2), 120-133. doi: 10.5731/ pdajpst.2015.006098.

Patiño, R., Silva, J. d., \& Pérez, J. (2011). Modelos de predicción de exigencias minerales para rumiantes. Revista Colombiana de Ciencia Animal, 3 (2), 344-365. 